\title{
CFD Analysis Of Multi-Phase Flow And Its Measurements
}

\author{
C Rajesh Babu ${ }^{1}$ \\ ${ }^{1}$ Mechanical Engineering Department, GITAM School of Technology, GITAM UNIVERSITY, Rudraram, \\ HYDERABAD-502329, India.
}

\begin{abstract}
Multiphase flow occurs when more than one material is present in a flow field and the materials are present in different physical states of matter or are present in the same physical state of matter but with distinct chemical properties. The materials present in multiphase flow are often identified as belonging to the primary or secondary phases. The primary phase is characterized as the phase that is continuous about, or enveloping of, the secondary phase. The secondary phase is thought to be the material that is distributed throughout the primary phase. Each phase present in multiphase flow may be either laminar or turbulent, which leads to a variety of potential flow regimes for multiple phases in the same channel. Project is based on two-phase flow and its measurement (water + air/vapor). This is frequently encountered in thermal and nuclear power plants, $R \& A / C$ and cryogenic applications, chemical industries and biotechnology etc., the arrangement of a vertical tube with two water inlets and three air inlets. By varying air and water flow rates following things are demonstrated and calculated:
\end{abstract}

Flow regime identification through visualization

Pressure drop measurement

The analysis carried out by the flow of air + water mixture using by Computational Fluid Dynamics (CFD) technique.

Keywords: Multiphase; Vertical tube; Two water inlets; Three air inlets; ANSYS-FLUENT

\section{Introduction}

Multiphase flows take place when several phases or components flow in the same space. The simpler case of two-phase flow denotes the simultaneous flow of two different phases. Two-phase flows can be liquid and gas, liquid and solid (e.g., slurries), or gas and solid (e.g. dusts, aerosols, etc). In energy technology, we are most often concerned with two-phase flows of a liquid and a gas. Liquid-gas mixtures of two different chemical components (e.g. air and water) are called two-component mixtures. Mixtures of a liquid and its vapor are called single-component flows. A particular case of single component flows are the flows of water and steam mixtures. Another categorization of flows is according the presence or absence of heat transfer: we speak of adiabatic or diabetic multiphase flows. In diabetic multiphase flows, we can have phase change: vaporization or condensation. Another important distinction is according to the flow direction: vertical, horizontal, or inclined. Flow direction is more important in multiphase flows where gravity may play a more important role in presence of large differences in the density of the phases; such differences can easily be of the order of 1000 (e.g., air water flow at atmospheric pressure). Finally, we speak of parallel or co-current flow when the two phases flow in the same direction and of counter-current flow when they flow in opposite directions (e.g., falling liquid and rising gas).The introduction to two-phase flow given below applies to one-dimensional two-phase flows in channels. Examples are the flow of steam and water in the tubes of a steam generator or around the fuel rod bundle of a boiling water reactor, the flow of petroleum and natural gas in a pipeline, the dispersed flow of steam and droplets at the exit of a turbine, etc. The analysis of single-phase is made easier if it can be established that the flow is either laminar or turbulent and whether any separation or secondary flow effect occurs. This information is equally useful in the study of gas-liquid flow. However, it is of greater importance in the latter case in topology or geometry of the flow. One of the difficulties in treating analytically two-phase flows lies in the presence of interfaces separating the two phases. It is only in a very restricted number of cases that the shape of the interfaces is roughly predictable: dispersed flow of spherical particles (bubbles or droplets) and smooth stratified or annular flow are such examples (Methods to predict the shape of interfaces in general, using CFD techniques, are still at their infancy). Flow regimes in vertical pipes can be seen in Figure 1 below: 

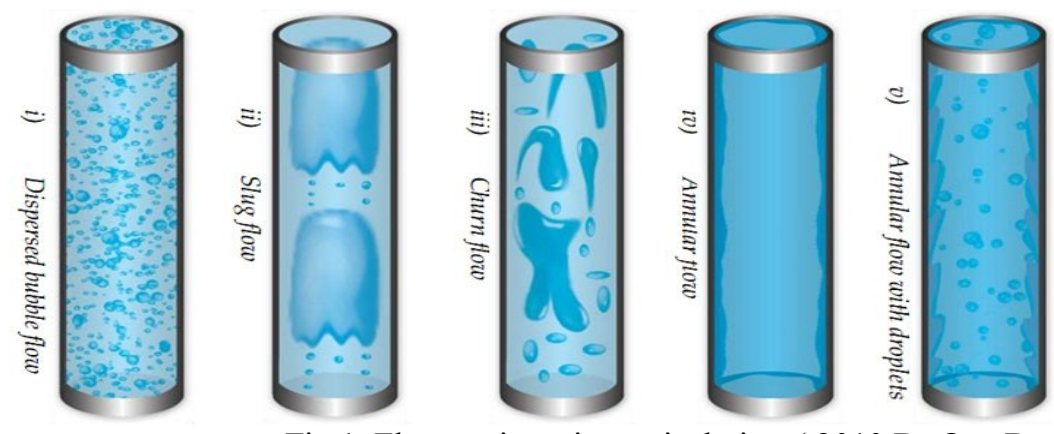

Fig.1. Flow regimes in vertical pipes( 2010 Dr.Ove Bratland)

\section{Methodology}

To analyze the two phase flow and to evaluate the performance, the basic steps involved in solving any CFD problem are as follows:

Identification of flow domain.

Geometry Modelling.

Grid generation.

Specification of boundary Conditions.

Selection of solver parameters and

Convergence criteria.

Results and post processing.

It is well-known to us that steam is a common two phase mixture that is encountered in most of the applications where water and vapor are the two phases i.e. water and air. So here we are going to allow both air and water mixture in a pipe. The material we have chosen is of acrylic resin. This is such kind of material that we can visualize the changes happening inside it.

Following dimensions:

Length $=2 \mathrm{~m}$

Outer diameter $=50 \mathrm{~mm}$

Inner diameter $=44 \mathrm{~mm}$

Shortly it can be termed as a $2 \mathrm{~m}$ and $2 "$ pipe

Two through holes (dia.8mm) are drilled at one end of the pipe at two diametrical ends and with an offset. These are for the water inlets. This pipe is inserted into a groove, which is a contour of the circular wall of the pipe, made on a base of same material. This is a 12" thickness acrylic sheet. The same material is chosen in order to form a perfect bondage in assembly which is done with the help of a solution (chloroform) thus applied. Three holes (dia.6mm) are drilled in this platform so that they form three vertices of an equilateral triangle with a circumcircle of $25 \mathrm{~mm}$ diameter to allow air nozzles. Air nozzles are small acrylic rods of $6 \mathrm{~mm}$ outer dia. acrylic rods with $1.2 \mathrm{~mm}$ hole drilled inside. Air is allowed from air compressor via pressure regulator. This controls the pressure with which air is allowed. Amount of air that is given is calculated with the help of specially designed air orifice meter. Thus regulated amount of air is passed into the pipe through air nozzles. Simultaneously, water from overhead tank is passed through water Rota meter and supplied to the two water inlets. These are done via various pipe fittings and flexible pipes. Thus air and water are allowed into the pipe creating a two phase flow mixture.

Fig. 2\&2a Experimental set-up drawn using AUTO CAD and Fabricated experimental setup 


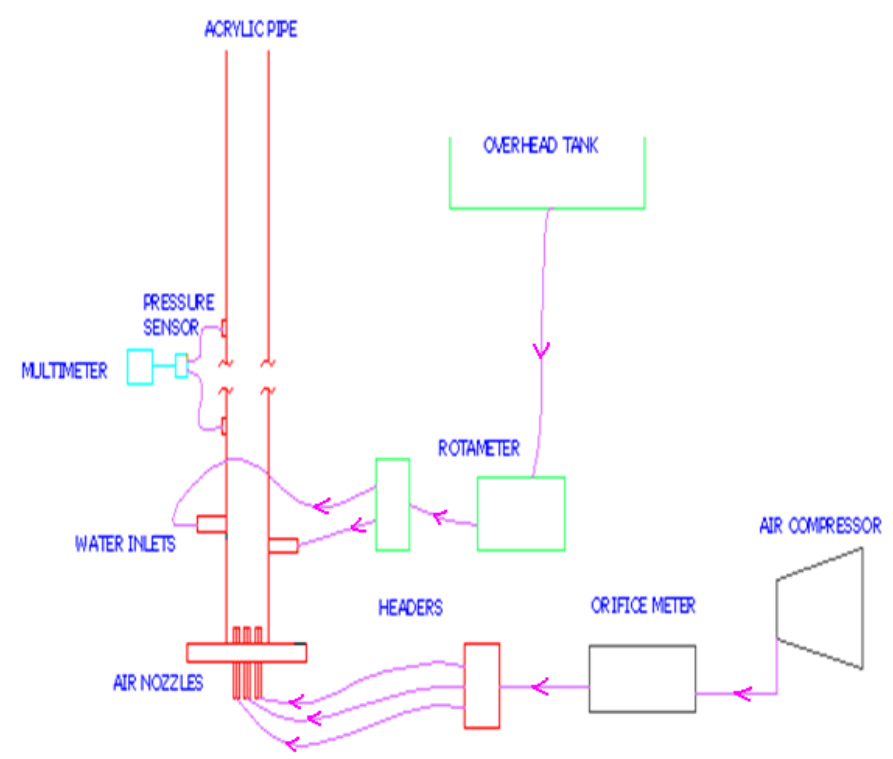

Fig.2.Experimental set-up drawn using AUTO CAD

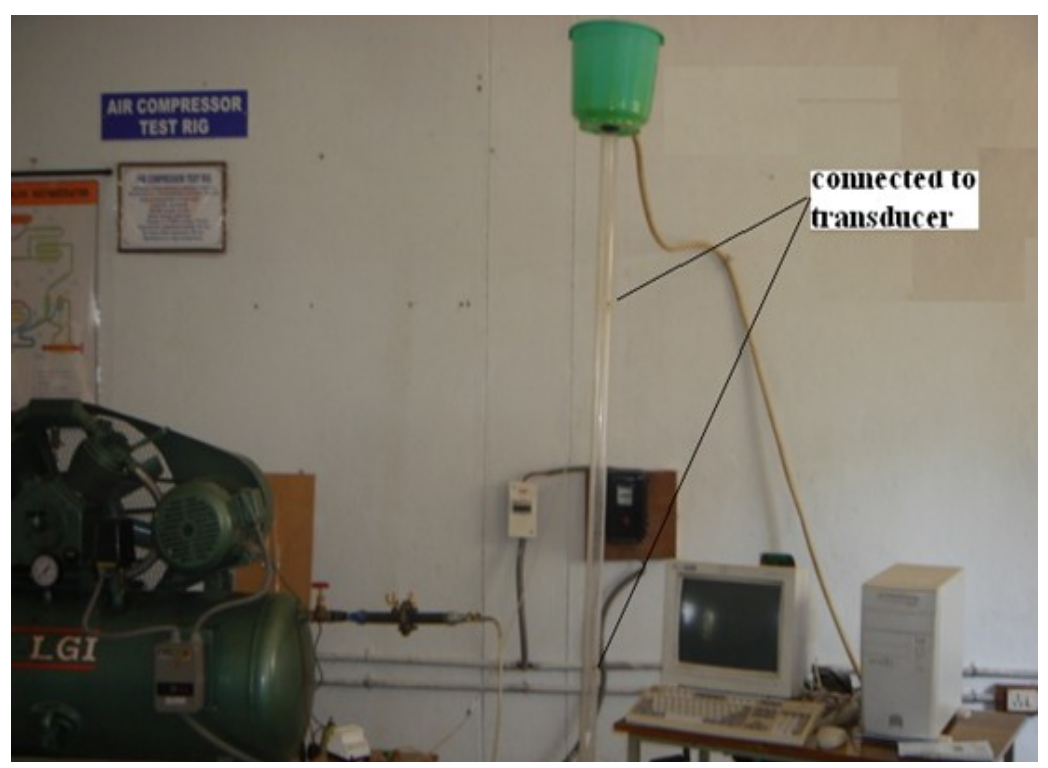

Fig .3. Fabricated experimental setup

This involves the preparation of data, such as nodal coordinates, connectivity information and boundary conditions. In the present context, a 2D diagram of the pipe is prepared. This is done as follows:

Table 1. Drawing the vertices i.e. nodal coordinate

\begin{tabular}{|l|l|l|}
\hline $\begin{array}{l}\text { Vertices } \\
\text { (nodes) }\end{array}$ & x-coordinate & y-coordinate \\
\hline 1 & 0 & 0 \\
\hline 2 & 0.020 & 0 \\
\hline 3 & 0.025 & 0 \\
\hline 4 & 0.047 & 0 \\
\hline 5 & 0.050 & 2 \\
\hline 6 & 0.024 & 2 \\
\hline 7 & 0.020 & 2 \\
\hline 8 & 0 & 2 \\
\hline
\end{tabular}


Table 2.Formation of edges

\begin{tabular}{|l|l|}
\hline Edges & Nodes \\
\hline 1 & 1,2 \\
\hline 2 & 2,3 \\
\hline 3 & 3,4 \\
\hline 4 & 4,5 \\
\hline 5 & 5,6 \\
\hline 6 & 6,7 \\
\hline 7 & 7,8 \\
\hline 8 & 8,1 \\
\hline
\end{tabular}

Face formation: A face is prepared connecting all edges.

Meshing the edges: Meshing is the process of dividing an edge into finite no. of parts. This is a process of discretization where the properties are studied at finite level. Here, the no. of parts that an edge is divided is noted

Table 3.Formation of meshing edges

\begin{tabular}{|l|l|}
\hline Edges & Nodes \\
\hline 1 & 1,2 \\
\hline 2 & 2,3 \\
\hline 3 & 3,4 \\
\hline 4 & 4,5 \\
\hline 5 & 5,6 \\
\hline 6 & 6,7 \\
\hline 7 & 7,8 \\
\hline 8 & 8,1 \\
\hline
\end{tabular}

Meshing the face: After meshing the edges, face is also meshed. The platform of the Gambit indicating the formation of mesh is shown as follows.Fig.3. The platform of the GAMBIT modeling indicating the formation of mesh

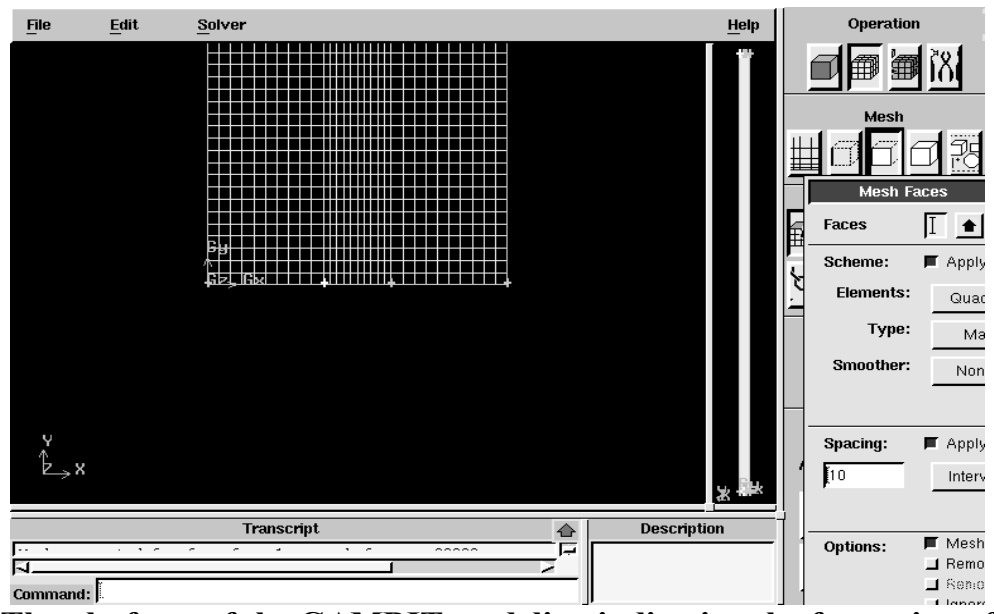

Fig.3. The platform of the GAMBIT modeling indicating the formation of mesh

\section{Formation of zones:}

Following are the zones that are defined in this modeling-

Zone 1 - Velocity inlet

Zone 2 - Wall

Zone 3 - Pressure outlet

Exporting the mesh:The mesh thus formed is saved as an '.msh' file and is exported. 'Fluent' is selected as the solver. 


\section{Methodology}

Here actual analysis is performed which involves fluid computations and the output will be in the form of numerical data.

The steps involved in the analysis are

1. Read the mesh file i.e. importing the exported file

2. Checking the grid

3. Defining the problem

- Model

- Materials

- Phases

- Operating conditions

- Boundary conditions

4. Selecting a numerical method

5. Initialize the problem

6. Write it to a file

7. Iterate the problem

Discretization scheme: Upwind ( $1^{\text {st }}$ order)

$\&$ High Resolution $\left(2^{\text {nd }}\right.$ order $)$

Time Scale Control: Auto Time Scale

Maximum Iterations: 1500

Residual Convergence criteria: RMS

Residual Convergence Target: 1E-5

Post-Processing: Here it accepts the results of analysis and generates tables, pictures etc. for proper interpretation of results after the solution are converged, and the contours of different properties along the length of the pipe are displayed. Graphs can also be plotted. The grid that is imported had been checked and displayed as follows in the fluent package:
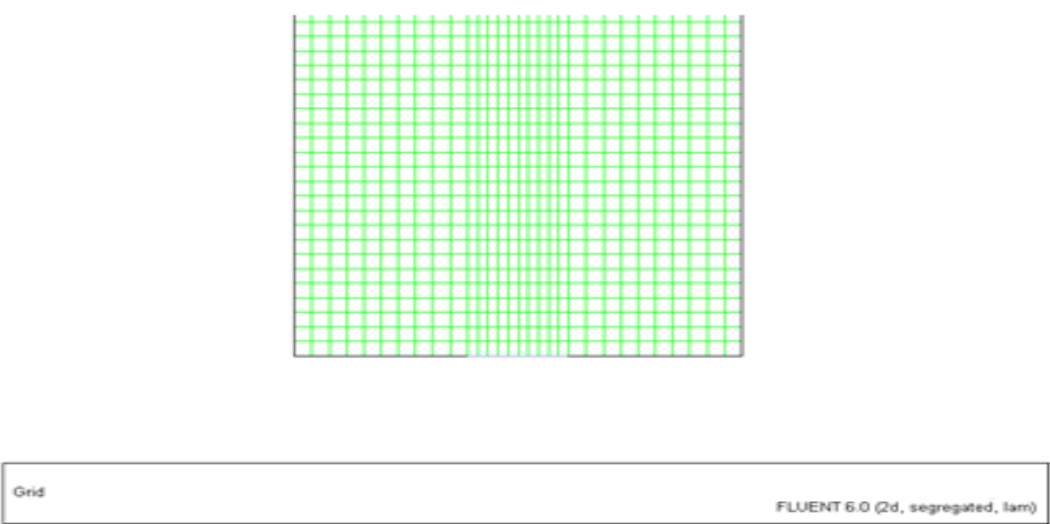

Fig.4.Bottom portion of the imported grid in FLUENT

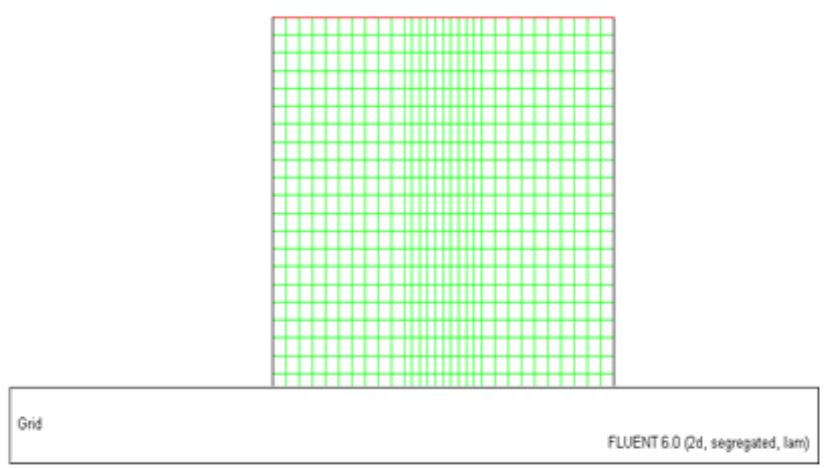

Fig.5.Top portion of the imported grid in FLUENT 
Air + water mixture is given at the inlet zone as velocity inlet zone. Air is introduced at a velocity of 26.6412 $\mathrm{m} / \mathrm{s}$ and water at a velocity of $\mathbf{0 . 6 6 2 7} \mathrm{m} / \mathrm{s}$.

The flow rates are: Water $-\mathbf{0 . 0 6} \mathbf{~ k g}$. $/ \mathbf{s}$ : Air $-\mathbf{0 . 0 0 4} \mathbf{~ k g . ~} / \mathbf{s}$

\section{Solutions}

The solution has converged after 1204 iterations. The velocities, dynamic pressure contours of different properties along the length of the pipe are displayed. Graphs can also be plotted.

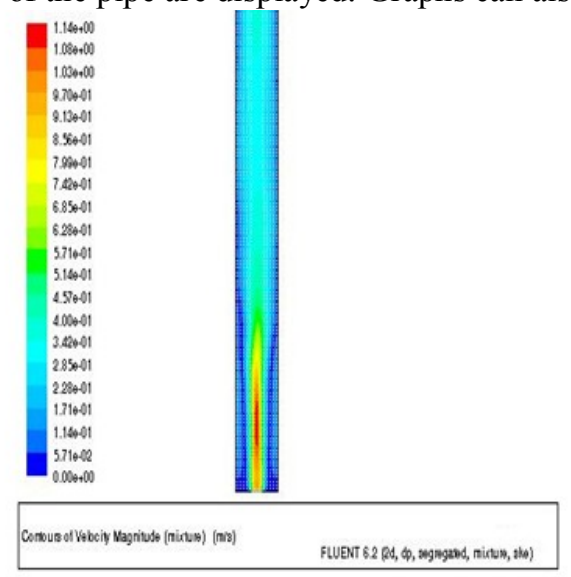

Fig.6.contours of velocity distribution along the pipe

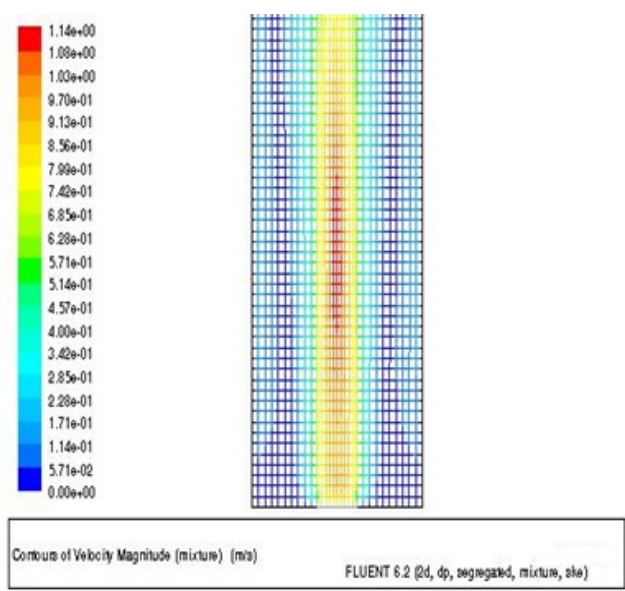

Fig.7.Contour of velocity distribution along the pipe

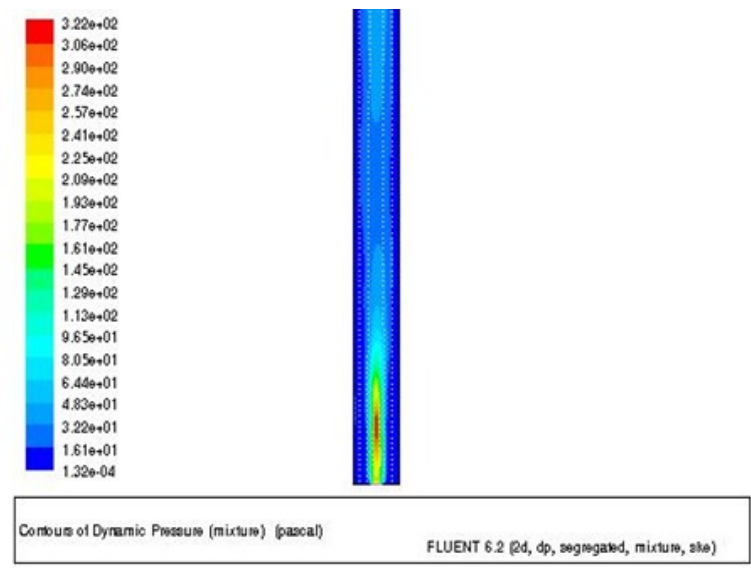

Fig.8.Contour of Dynamic pressure distribution along the pipe 


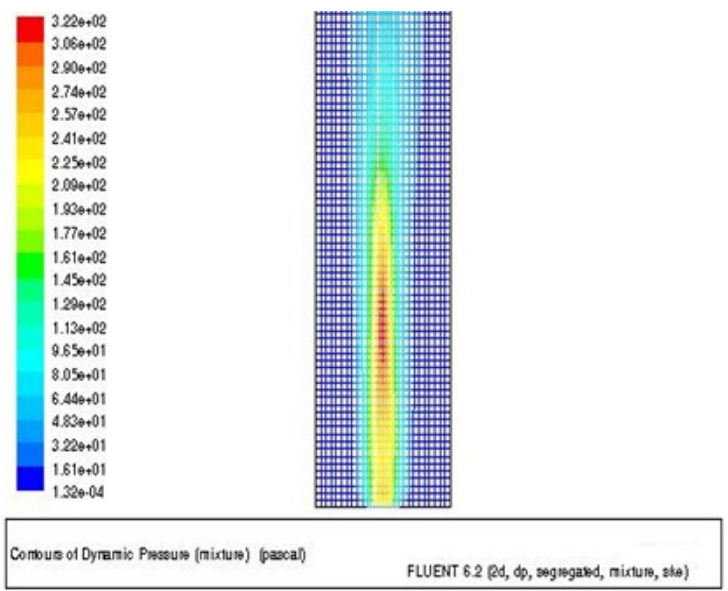

Fig.9.Contour of pressure distribution along the pipe

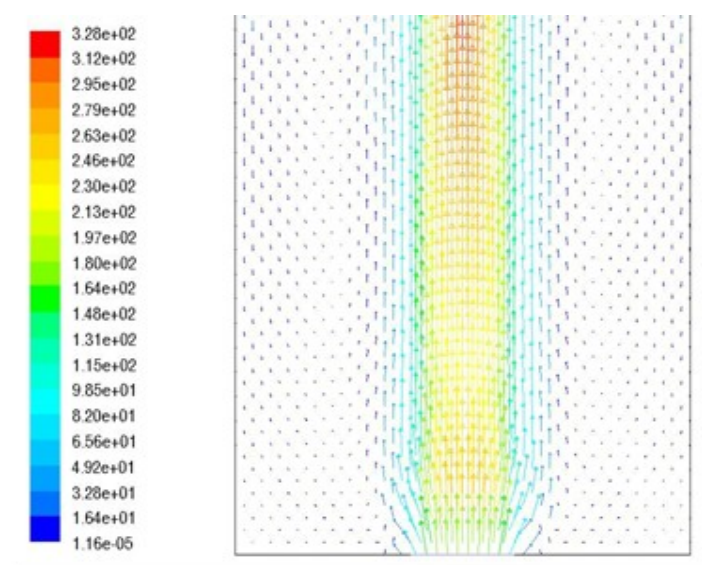

Velocity Vectors Colored By Dynamic Preosure (mixture) (pascal)
FLUENT 6.2 (2d, dp, segregated, mixture, oke)

Fig.10.Pressure Vectors along the pipe

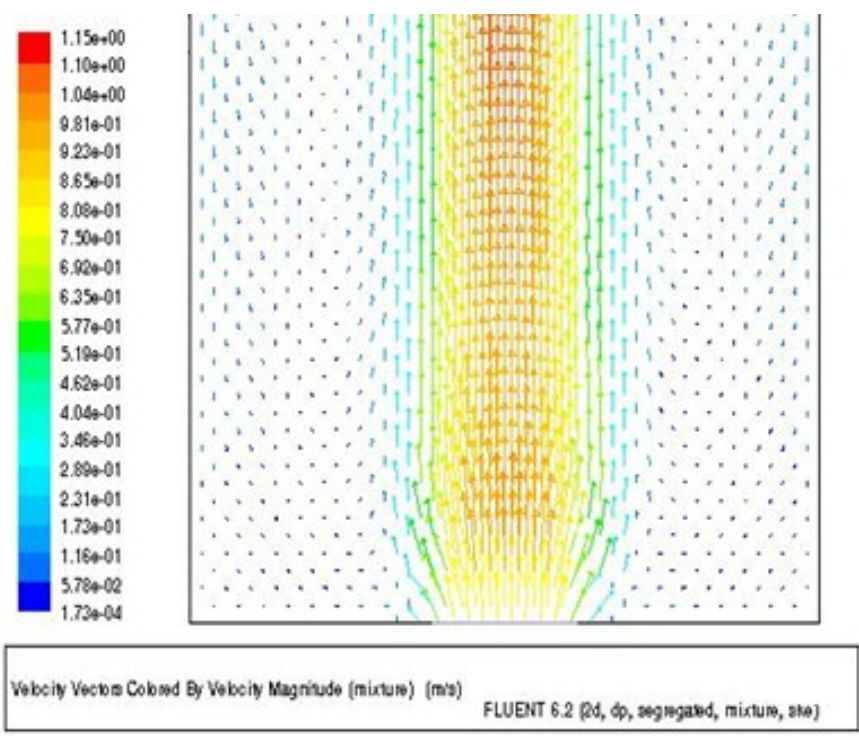

Fig.11.Velocity Vectors along the pipe 


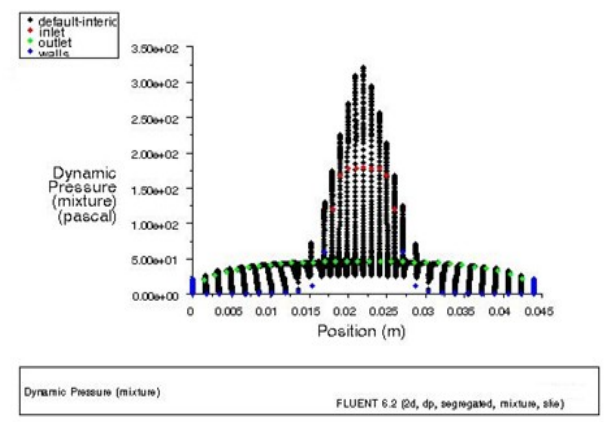

Fig.12. Plot of pressure distribution along the pipe

\section{Results And Discussions}

With this built experimental apparatus, where air + water mixture is flowing, by controlling the air flow rate, we can visualize different flow regimes that are previously defined. Also arranged a conductivity probe that is inserted into the pipe which is connected to an electronic circuit which is further connected to voltage detector that detects random variations of the phases (air and water) in terms of the variation of voltage. This is due to different conduction properties of air and water. The variation which can be visualized tells us the amount of air and water that is present at that point and the time for which the phase lasts there. Flow regimes in vertical pipes are shown below .

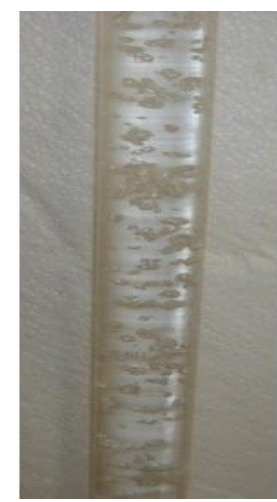

i) Bubbly flow

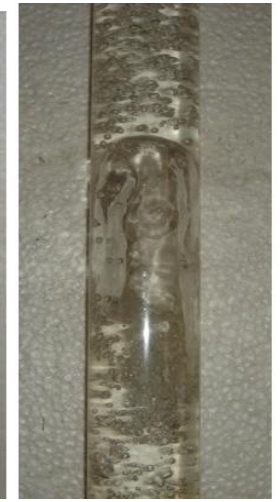

ii) Plug flow

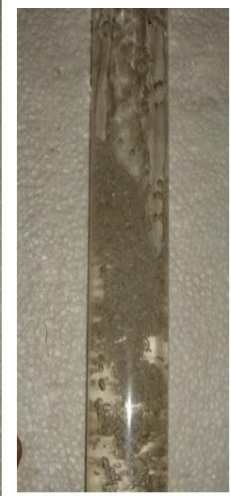

iii) Churn flow

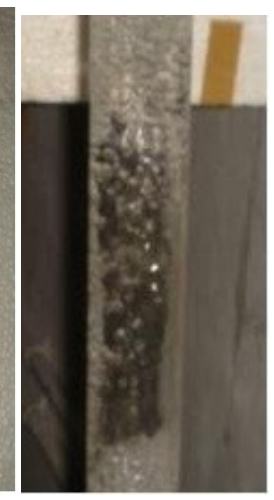

iv)Annular flow

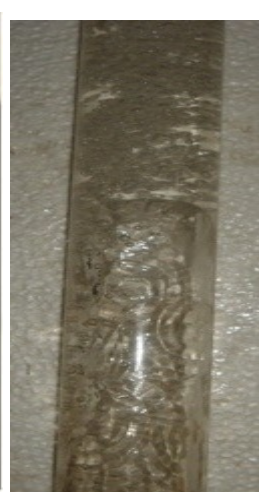

v) Wispy annular flow

Fig.13. Flow regimes in vertical pipes

\section{Conclusions}

This is done by means of an analysis of the sensitivity to variations of the main parameters such as velocity, pressure etc.., and also by comparing the results with two sets of original experimental data on air and water critical flows. Finally, the model is tested in turbulent conditions.

- Demonstration of two phase flow, different flow regimes, variations that occur in amount of air and water at a particular point

- $\quad$ Pressure drop and Velocity variations

- CFD Analysis of the flow

\section{References}

[1]. Aug. 1969, Symposium on Two-Phase Flow Instrumentation presented at $11^{\text {th }}$ National ASME / A.I.Ch.E. Heat Transfer Conf. Minneapolis, Minnesota.

[2]. Collier, J.G., and Thome, J.R., 1994, "Convective Boiling and Condensation," 3rd edition, Oxford Science Publications, Clarendon Press.

[3]. Collier, J.G., and Hewitt, G.F., 1966, “Experimental Techniques in Two-phase Flow,” British Chemical Engineering, II, 12, 15261531.

[4]. G.gopalkrishnan, and D, PrithviRaj, Treatise on Turbo machines, Scitech publications, Chennai, 2002.

[5]. Hewitt, G.F., 1978, Measurement of Two-Phase Flow Parameters, Academic Press, London.

[6]. Hestroni, G., Handbook of Multiphase Systems, Hemisphere, Washington, 1982.

[7]. Mayinger, F Stroemung und Waermeubergang in Gas-Fluessigkeits-Gemischen, Springer-Verlag, Wien, New York, 1982,

[8]. Patankar, Suhas v, Numerical heat transfer and Fluid flow. Hemisphere publications, USA.1980.

[9]. H.K.versteeg and W.Malalasekera, An introduction to CFD the Finite volume method, Pearson education limited,2005

[10]. Wallis, G. B., One-Dimensional Two-Phase Flow, McGraw Hill Book Company, New York, 1969. 